\title{
L'économie pastorale face aux incertitudes*
}

\author{
Le salariat au Ferlo (Sahel sénégalais)
}

Abdrahmane Wane $1,2,3$

Véronique Ancey ${ }^{1,2,3}$

Ibra Touré $1,2,3$

Samba Ndiobène Kâa

Astou Diao-Camara ${ }^{3}$

${ }^{1}$ Cirad

UR Systèmes d'élevage

Délégation régionale du Cirad

BP 6189 Dakar-Etoile

99999 Dakar

Sénégal

<abdrahmane.wane@cirad.fr>

$<$ veronique.ancey@cirad.fr>

<ibra.toure@cirad.fr>

${ }^{2}$ Cirad

UR Systèmes d'élevage

Montpellier

F-34398 France

3 Isra-LNERV

PPZS (Pôle pastoral en zones sèches)

BP 2057

Dakar-Hann

Sénégal

<sougoufarafr@yahoo.fr>

${ }^{4}$ SEN AGRO BUSINESS

Cabinet de Consulting

Boulal - Dahra

Sénégal

<sambandiobene@yahoo.fr>

\begin{abstract}
Résumé
Face aux chocs et aux incertitudes structurelles au Sahel, l'élevage transhumant a prouvé sa résilience au moyen de stratégies traditionnelles: mobilité et diversification. Aujourd'hui, $25 \%$ des exploitations du Ferlo utilisent de la main-d'œuvre salariée, réorganisant les tâches habituellement pratiquées à l'échelle familiale. Cet article aborde le salariat comme une stratégie qui répond aux incertitudes internes (disponibilité de maind'œuvre) et externes (diversification des espèces et rareté des ressources naturelles) et contribue à la gestion continue du troupeau, pilier central de la sécurité alimentaire.
\end{abstract}

Mots clés : changement structurel ; main d'œuvre ; pastoralisme ; Sahel.

Thèmes : économie et développement rural ; productions animales.

\section{Abstract \\ Pastoral economy facing up to uncertainties. Wage labour in the Ferlo (Senegalese Sahel)}

In the Sahelian context of shocks and structural uncertainties, transhumant breeding has proved its resilience by relying on traditional strategies such as mobility and diversification. Today, 25 percent of the investigated encampments of Ferlo have used a salaried workforce, renewing the delegation of tasks usually handled at the family scale. This article shows wage-labour as a strategy for copying with uncertainties inside the family (workforce availability) and outside (diversification of species and natural resource scarcity) to ensure continuity of herd management as the central pillar of pastoral food security.

Key words: manpower; pastoralism; Sahel; structural change.

Subjects: animal productions; economy and rural development.
A u Sahel, le troupeau demeure le principal patrimoine des pasteurs transhumants. Sa gestion requiert plusieurs tâches exigeant parfois le recours à de la main-d'œuvre salariée. L'arbitrage entre le travail au sein et hors de l'exploitation ${ }^{1}$ a suscité de nombreux travaux mais très peu ont porté sur la demande de travail salarié des exploita- tions agricoles (Blanc et al., 2008). De plus, ces rares contributions ont plutôt étudié les exploitations agricoles de type capitaliste (Vanderman et al., 1991). Cet article aborde la demande de travail salarié dans les exploitations pastorales du Ferlo où les décisions de production et de vente sont généralement déconnectées des impératifs du marché (Wane

\footnotetext{
* Pour citer cet article: Wane A, Ancey V, Touré I, Kâ SN, Dio-Camara A. L'économie pastorale face aux incertitudes - Le salariat au Ferlo (Sahel sénégalais). Cah Agric 2010 ; 19 : 359-65. DOI : 10.1684/agr.2010.0427

${ }^{1}$ Dans la littérature, l'expression "exploitation agricole " est le plus souvent utilisée. Au Ferlo, l'unité de production, de résidence et de gestion du troupeau est désignée par le terme " campement ". Ces deux termes représentent la même chose et sont indifféremment utilisés tout au long de cette étude.
} 
et al., 2010). Sous certaines conditions, ce salariat traduit un trait économique inscrit dans les objectifs structurels des pasteurs : sécurité alimentaire (Ancey et Monas, 2005) et continuité de la production et de l'unité familiale (Wane et al., 2009). Cette étude propose un dispositif de collecte d'informations adapté à la vie pastorale ; elle analyse le cadre théorique et les hypothèses sous-jacentes à l'identification des déterminants du recours à de la main-d'œuvre salariée au Ferlo et discute de ses caractéristiques comme une stratégie opportuniste face aux incertitudes.

\section{Dispositif de collecte d'informations}

Le Ferlo recouvre environ un tiers du territoire national avec une superficie de $67610 \mathrm{~km}^{2}$. Délimitée au nord par la vallée du fleuve Sénégal et au sud par le front du Bassin arachidier, cette zone bénéficie d'un climat caractérisé par des précipitations concentrées sur 2 à 3 mois d'été et des moyennes annuelles inférieures à $200 \mathrm{~mm}$ à l'extrême nord et supérieures à $550 \mathrm{~mm}$ au sud (figure 1). La principale activité de valorisation de cet espace aride est l'élevage pastoral exercé de façon extensive. L'étude du système de production pastoral au Ferlo, activité qualifiée d' informelle " car déficiente en données primaires, a requis des approches quantitatives et qualitatives, avec des questionnaires semi-fermés et des guides d'entretien. Le choix des sites et l'échantillonnage des campements à enquêter ont été effectués pour qu'ils soient représentatifs de la diversité socio-écologique de la région et de la mobilité des pasteurs (Wane et al., 2009) (figure 1). L'unité d'enquête est le campement (Wane et al., 2009). Les principaux répondants aux enquêtes sont les chefs de campements. Les données tirées des questionnaires sont complétées par des entretiens dans 9 campements pour cerner les motivations des pasteurs et les déterminants qualitatifs du salariat. À partir de critères validés avec un panel de pasteurs, des seuils distinguent les très gros, gros, moyens et petits éleveurs parmi les 914 campements recensés. Un échantillon de 149 campements a fourni des données collectées sur plusieurs exploitations pastorales à la même période (tableau 1). L'essentiel des recettes pastorales provient des ventes d'animaux (Wane et al., 2010) : les productions animales demeurent capitales dans l'économie familiale. Notre hypothèse centrale est que leur sécurisation est une priorité. Cette sécurisation passe par l'accès aux ressources. La mobilité constitue la principale stratégie de gestion des troupeaux au Ferlo qui s'organise à $98 \%$ du temps autour de la pâture, de l'abreuvement, de la traite et de la transhumance.

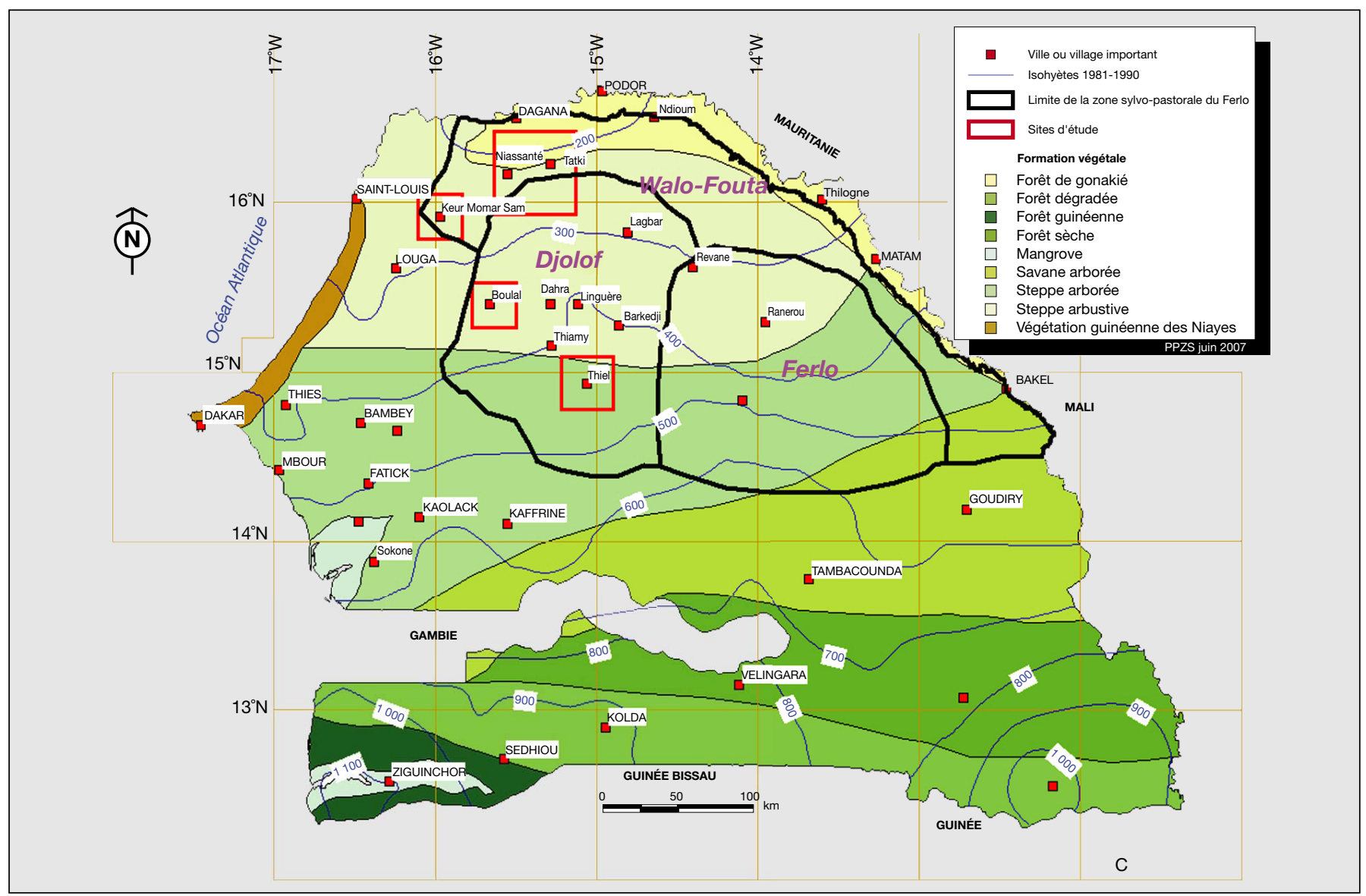

Figure 1. Carte des sites d'échantillonnage.

Figure 1. Map of the sampling sites.

Source : Touré, PPZS, 2007. 
Tableau 1. Caractéristiques de l'échantillonnage des campements.

Table 1. Caracteristics of the encampment sampling.

\begin{tabular}{|c|c|c|c|c|c|c|}
\hline \multirow[t]{2}{*}{ Sites } & \multirow{2}{*}{$\begin{array}{c}\text { Nombre } \\
\text { de campements }\end{array}$} & \multirow[t]{2}{*}{ En \% } & \multicolumn{3}{|c|}{ Échantillons * } & \multirow{2}{*}{$\begin{array}{l}\text { Échantillon } \\
\text { pondéré }\end{array}$} \\
\hline & & & théorique $^{1}$ & théorique $^{2}$ & réaliste $^{3}$ & \\
\hline Boulal & 268 & 29 & 144 & 79 & 44 & 57 \\
\hline Keur M. Sarr & 174 & 19 & 94 & 51 & 28 & 37 \\
\hline Niassanté & 204 & 22 & 110 & 60 & 33 & 43 \\
\hline Tatki & 105 & 11 & 57 & 31 & 17 & 22 \\
\hline Thiel & 163 & 18 & 88 & 48 & 27 & 35 \\
\hline Total & 914 & 100 & 493 & 271 & 149 & 195 \\
\hline
\end{tabular}

${ }^{1} \mathrm{e}=3 \%$; intervalle de confiance (ic) $=95 \% ; p=50 \%$

$2 \mathrm{e}=5 \%$; ic $=95 \% ; p=50 \%$

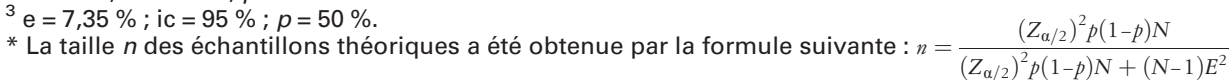

En moyenne, $25 \%$ des campements salarient de la main-d'œuvre externe. Trois groupes de sites se distinguent spatialement. Une proportion élevée de recrutements concerne Keur Momar Sarr, zone de périmètres agricoles irrigués, et Tatki, vers la vallée du fleuve Sénégal (respectivement 39 et $35 \%$ ). Une proportion médiane se constate à Boulal, près de la ville de Dahra, principal marché de bétail de la zone pastorale et Thiel situé sur le front agricole arachidier (respectivement 23 et $26 \%$ ). Et une faible proportion, moins de $10 \%$, se note à Niassanté, au coeur de la zone pastorale avec de faibles possibilités de diversification et une disponibilité notable de main-d'œuvre de $36 \%$.

L'approche par catégories d'éleveurs montre que $69 \%$ des très gros, $16 \%$ des gros et $15 \%$ des moyens de campements d'éleveurs salarient de la main-d'œuvre externe et que les petits n'en ont pas (figure 2). Les exploitations sont monosalariées à $59 \%$; seuls $8 \%$ des employeurs déclarent 5 recrues et plus.

\section{Aspects théoriques du salariat agricole et modélisation au Ferlo}

Les concepts de salariat agricole reflètent différents types d'arbitrages internes et externes: d'où l'utilité de délimiter nos cadres d'analyse et de préciser nos hypothèses de travail.

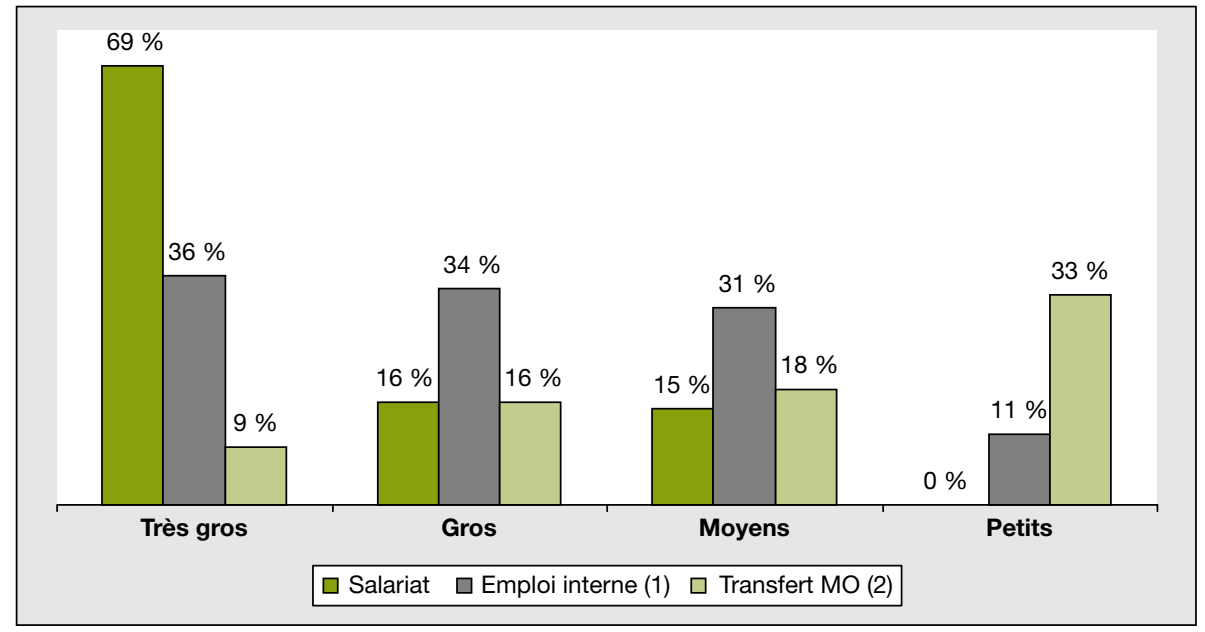

Figure 2. Salariat, emploi interne et externe par catégorie d'éleveurs.

Figure 2. Wage labour, internal and external employment by category of stockbreeders.

Source: auteurs sur données d'enquêtes.

(1) Le taux d'emploi interne est le rapport entre le nombre d'individus de l'exploitation déclarant travailler en interne et la population totale de l'exploitation.

(2) Le taux de transfert de main-d'œuvre est le rapport entre le nombre d'individus de l'exploitation déclarant travailler dans des activités externes rémunératrices et la population totale de l'exploitation.

\section{Le salariat agricole : résultat d'arbitrages internes et externes}

L'étude des exploitations agricoles distingue les exploitations de type capitaliste et les exploitations familiales (Chayanov, 1923). Les premières poursuivent une logique de maximisation des profits et interviennent sur les marchés, pilotes de leurs décisions de production et de vente. Les secondes ont une logique de maximisation du bien-être collectif recouvrant parfois des visées non monétaires et mènent des activités compatibles avec les buts du chef de l'exploitation (Gasson et al., 1988) ; elles s'inscrivent dans le corpus théorique du "ménage producteur-consommateur " conceptualisé par Chayanov (1923).

De nombreux travaux insistent sur les arbitrages entre le travail au sein et hors de l'exploitation. Ils traitent de la totalité du travail salarié et font abstraction de l'horizon temporel en supposant une substituabilité parfaite entre travail salarié permanent et saisonnier (Lass et Gempesaw, 1992; Kimhi, 2001), hypothèse contestée par d'autres (Blanc et al., 2008). Les déterminants de l'offre de 
Tableau 2. Variables potentiellement influentes dans le recours au salariat.

Table 2. Potentially influential variables in the use to wage labour.

\begin{tabular}{|c|c|c|c|}
\hline Catégories & Modalités & Variables & Signes attendus \\
\hline \multirow{4}{*}{$\begin{array}{l}\text { Nombre et degré d'implication } \\
\text { des individus du campement }\end{array}$} & Nombre de ménages & Men & - \\
\hline & Nombre d'individus dans la gestion des troupeaux & lges & - \\
\hline & Taux de transfert de main-d'œuvre & Thor & - \\
\hline & Taux d'emploi interne & Empi & - \\
\hline \multirow{6}{*}{$\begin{array}{l}\text { Genre et classe d'âge } \\
\text { des individus du campement }\end{array}$} & Nombre d'hommes & Hom & - \\
\hline & Nombre de femmes & Fem & + \\
\hline & Nombre d'enfants (moins de 10 ans) & Enf & + \\
\hline & Nombre d'adolescents (entre 10 et 18 ans) & Ado & - \\
\hline & Nombre d'adultes (entre 18 et 50 ans) & Adu & - \\
\hline & Nombre de vieux (plus de 50 ans) & Vieux & + \\
\hline \multirow{3}{*}{$\begin{array}{l}\text { Composition du troupeau } \\
\text { en espèces }\end{array}$} & Nombre de bovins & Bov & + \\
\hline & Nombre d'ovins & Ovi & + \\
\hline & Nombre de caprins & Cap & - \\
\hline Activités alternatives & $\begin{array}{l}\text { Potentiel de diversification dans activités } \\
\text { génératrices de revenus }\end{array}$ & Div & + \\
\hline
\end{tabular}

travail externe sont abordés à travers ses liens avec la variation ou la stabilisation du revenu agricole dans les exploitations de type capitaliste (Corsi et Findeis, 2000 ; Lass et Gempesaw, 1992; Mishra et Sandretto, 2002). Toutefois, dans les exploitations à ressources limitées, l'offre de travail externe est liée à la faiblesse du revenu plutôt qu'à sa variation (Vergara et al., 2004).

Plus généralement, la faiblesse et l'instabilité des rendements agricoles, la saisonnalité des récoltes, le déficit d'irrigation, les sécheresses, l'accès limité aux crédits et les contraintes foncières incitent les ménages agricoles à offrir du travail à l'extérieur (Reardon et al., 1992). D'autres déterminants concernent le nombre, la taille des ménages agricoles et leur capacité de diversification économique (Woldehanna et Oskam, 2001).

\section{Cadre conceptuel, hypothèses de recherche, et modélisation}

Notre analyse du salariat au Ferlo cherche à prédire, à travers une régression logistique dichotomique, la décision des chefs de campements de recourir à de la main-d'œuuvre salariée. Cette décision est supposée être sensible aux caractéristiques socio-économique, démographique et zootechnique des campements (tableau 2).
Hypothèse 1 :

le nombre et le degré d'implication des individus influencent négativement cette décision

En première analyse, le travail dans les exploitations pastorales repose prioritairement sur les ressources familiales. Ainsi, les modalités à influence négative sur le recours à de la main-d'œuvre salariée sont :

- le nombre de ménages (Men) de l'exploitation, unités de consommation ; - le nombre d'individus résidents s'occupant de la gestion des troupeaux (Iges) ; - le taux d'emploi interne (Empi) ${ }^{2}$;

- le taux de transfert de main-d'œuvre (Thor) $)^{3}$.

\section{Hypothèse 2 : \\ la composition populationnelle influence cette décision}

La spécialisation par tâches au sein des exploitations - gestion et conduite des troupeaux par les hommes et travaux ménagers et gestion des produits laitiers par les femmes - peut amener la compo-

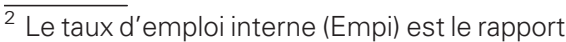
entre le nombre d'individus de l'exploitation déclarant travailler en interne et la population totale de l'exploitation.

${ }^{3}$ Le taux de transfert de main-d'œuvre (Thor) est le rapport entre le nombre d'individus de I'exploitation déclarant travailler dans des activités externes rémunératrices et la population totale de l'exploitation.
}

sition en genre et la structure des âges de l'exploitation à influencer la demande de travail externe.

Les modalités à influence négative sont :

- le nombre d'hommes résidant dans l'exploitation (Hom) ;

- le nombre d'adolescents entre 10 et 18 ans (Ado) ;

- le nombre d'adultes entre 19 et 50 ans (Adu).

Les modalités à influence positive sont :

- le nombre de femmes (Fem) ;

- le nombre d'enfants de moins de 10 ans (Enf) ;

- le nombre d'individus de plus de 50 ans (Vieux).

\section{Hypothèse 3 :}

la composition du troupeau en espèces influence cette décision

Le salariat porte sur les tâches de gestion et de conduite des troupeaux mais toutes les espèces ne sont pas également concernées.

Les modalités influençant négativement la décision de recruter sont le nombre de caprins (Cap) - espèce ne faisant pas l'objet d'une surveillance particulière.

Les modalités l'influençant positivement sont le nombre de bovins (Bov) - espèce prestigieuse à forte valeur patrimoniale et le nombre d'ovins (Ovi) - espèce très peu autonome, nécessitant une surveillance étroite en période de mobilité. 
Hypothèse 4 :

la diversification économique (Div) affecte positivement cette décision?

Pour éviter tout problème de multicolinéarité, deux modèles sont testés : - l'un tenant compte du "genre ":

$$
\begin{aligned}
\text { Salariat }= & C+\beta_{1} \text { Men }+\beta_{2} \text { Iges }+\beta_{3} \text { Thor } \\
& +\beta_{4} \text { Empi }+\beta_{5} \text { Hom }+\beta_{6} \text { Fem } \\
& +\beta_{7} \text { Bov }+\beta_{8} \text { Ovi }+\beta_{9} \text { Cap } \\
& +\beta_{10} \text { Div }
\end{aligned}
$$

Où :

$\mathrm{C}=$ constante $; \quad \beta_{i}:$ coefficient de la variable i dans le modèle.

- l'autre tenant compte de la "classe d'âge ":

$$
\begin{aligned}
\text { Salariat }= & C+\beta_{1} \text { Men }+\beta_{2} \text { Iges }+\beta_{3} \text { Thor } \\
& +\beta_{4} \text { Empi }+\beta_{5} \text { Enf }+\beta_{6} \text { Ado } \\
& +\beta_{7} \text { Adu }+\beta_{8} \text { Vieux }+\beta_{9} \text { Bov } \\
& +\beta_{10} \text { Ovi }+\beta_{11} \text { Cap }+\beta_{12} \text { Div }
\end{aligned}
$$

Où :

$\mathrm{C}=$ constante $; \beta_{i}:$ coefficient de la variable i dans le modèle.

La taille de l'échantillon et le nombre de variables font que le pseudo $\mathrm{R}^{2}$ de Nagelkerke d'au moins $42 \%$ est significatif (Hair et al., 1998). Ainsi, le choix des modalités explicatives du recours à de la main-d'œuvre salariée, s'avère pertinent (tableau 3).

\section{Résultats}

\section{et discussions}

\section{sur le recours \\ à de la main-d'œuvre \\ salariée}

Nos résultats accréditent la plupart des hypothèses initiales. Dans les deux modèles, le recours à de la main-d'œuvre salariée est très significativement déterminé (au seuil de $1 \%$ ) par le nombre de ménages (Men), le nombre d'ovins (Ovi) et le taux de transfert de main-d'œuvre (Thor). Ces modalités constituent respectivement des propensions à recourir à de la maind'œuvre salariée qui, en moyenne, baissent de 38,5\% par ménage supplémentaire dans l'exploitation et de 95,5\% par unité de transfert de main-d'œuvre, mais augmentent de 0,85\% par ovin supplémentaire dans le troupeau. Plus spécifiquement, le nombre de femmes (Fem) dans le campement est une modalité significative (au seuil de 5\%) dans le modèle "genre " et indique que la propension à recourir à de la main-d'œuvre salariée augmente de 13,1\% par femme supplémentaire dans l'exploitation. Le nombre de personnes âgées (vieux) est relativement significatif (au seuil de
10 \%) dans le modèle "classe d'âge " et montre que la propension à recourir à de la main-d'œuvre salariée augmente de 36,2 \% par personne âgée supplémentaire dans l'exploitation.

Le salariat est une stratégie d'ajustement des employeurs du Ferlo aux incertitudes sur la continuité de la production pastorale : disponibilité de main-d'œuvre familiale et variations climatiques. Deux situations extrêmes coexistent. Les "petits" éleveurs ne recrutent pas de salariés et constituent un réservoir de maind'œuvre (33\%), notamment pour les autres catégories d'éleveurs. Les nécessités économiques les poussent à s'éloigner des tâches de gestion de leurs petits cheptels. Ce comportement s'explique par la faiblesse de leurs revenus, la présence d'individus dotés d'un certain savoirfaire pastoral et potentiellement disponibles pour d'autres exploitations, leur consommation comparativement plus frugale incitant la recherche d'autres sources de revenus. Le salariat demeure très présent chez les "très gros " éleveurs (69\%), chez qui il y a peu de transfert de main-d'œuvre (9\%). Ils savent saisir les opportunités économiques de leur envi-

\begin{tabular}{|c|c|c|c|c|c|c|}
\hline Type de modèle & Variables & B & E.S. & $\operatorname{Exp}(B)$ & $\begin{array}{c}\text { Odds ratios } \\
{[\operatorname{Exp}(B)-1] * 100} \\
(\%)\end{array}$ & $\begin{array}{c}R^{2} \text { de Nagelkerke } \\
(\%)\end{array}$ \\
\hline \multirow[t]{6}{*}{ Modèle " genre " } & Men*** & $-0,476$ & 0,171 & 0,621 & $-37,9$ & 42,0 \\
\hline & Fem** & 0,123 & 0,061 & 1,131 & 13,1 & \\
\hline & Thor*** & $-2,975$ & 1,146 & 0,051 & $-94,9$ & \\
\hline & Bov** & 0,012 & 0,006 & 1,012 & 1,2 & \\
\hline & Ovi ${ }^{* * *}$ & 0,009 & 0,003 & 1,009 & 0,9 & \\
\hline & Cte & 3,042 & 2,228 & 20,956 & - & \\
\hline \multirow{6}{*}{$\begin{array}{l}\text { Modèle } \\
\text { " classe d'âge " }\end{array}$} & Men*** & $-0,494$ & 0,182 & 0,610 & $-39,0$ & 42,4 \\
\hline & Vieux* & 0,309 & 0,168 & 1,362 & 36,2 & \\
\hline & Bov** $^{*}$ & 0,014 & 0,006 & 1,014 & 1,4 & \\
\hline & Ovi*** & 0,008 & 0,003 & 1,008 & 0,8 & \\
\hline & Thor*** & $-3,213$ & 1,177 & 0,040 & $-96,0$ & \\
\hline & Cte & 3,719 & 2,274 & 41,222 & - & \\
\hline
\end{tabular}
ronnement tout en restant attachés à l'élevage. Ils disposent de gros revenus provenant essentiellement de l'économie pastorale mais aussi d'autres sources. Ces revenus leur permettent de recruter

\section{Tableau 3. Influence des variables retenues sur la probabilité de recrutement.}

Table 3. Influence of the variables on the probability of recruiting.

*** significatif au seuil de $1 \%$, ** significatif au seuil de $5 \%$, significatif au seuil de $10 \%$.

B : coefficients traduisant les relations entre les variables indépendantes et la variable dépendante ; E.S : erreurs standard associées aux coefficients. Le pseudo $\mathrm{R}^{2}$ de Nagelkerke, version ajustée de celui de Cox \& Snell, est préféré car plus proche de la réalité.

Les variables non significatives n'apparaissant pas dans ce tableau. 
des salariés pour leurs importants cheptels. Chez tous les éleveurs exceptés chez les "petits", coexiste le recours, à des degrés divers, à de la main-d'œuvre salariée, à de la main-d'œuvre familiale et au transfert de main-d'œuvre. Cette situation illustre les multiples arbitrages au sein des exploitations entre l'offre de travail externe et interne. Les choix à arbitrer sont d'autant plus affirmés que le revenu est faible. La main-d'œuvre disponible est répartie en interne pour les besoins de l'exploitation et en externe en contrepartie d'une rétribution monétaire ou non dans d'autres exploitations, voire dans des activités économiques alternatives, souvent le commerce (figure 2). Cette situation est exacerbée chez les "petits " éleveurs dont une infime partie de la main-d'œuvre disponible gère en interne le troupeau et une majorité offre du travail en externe.

Un autre déterminant spécifique du salariat au Ferlo concerne les variations climatiques. Les régimes de mobilité constituent une adaptation des pasteurs aux conditions environnementales et une sécurisation du cheptel (Marshall et Hildebrand, 2002) au-delà des controverses sur les causes de la dégradation des ressources privilégiant les facteurs tantôt anthropiques (Charney, 1975), tantôt climatiques (Scoones, 1999). Au Ferlo, cette mobilité provoque un recrutement souvent saisonnier ; dans environ deux tiers des campements recruteurs, le salarié est engagé pour la transhumance. Près de $87 \%$ des éleveurs parlant du recrutement comme d'une nécessité pour la continuité de la production ont aussi évoqué la pénibilité de l'exécution des tâches liées à la transhumance.

De façon plus générale, le recours à de la main-d'œuvre salariée dans le contexte informel d'embauche sans contrat écrit du Ferlo, incite à relire des études théoriques fondées sur des situations où le salariat est la forme dominante du travail et le contrat écrit la pièce centrale, quoiqu'insuffisante, de stabilisation de la relation salariale. Des incertitudes sur la production marchande peuvent provenir de la qualité et des comportements du salarié après le recrutement. Dès lors, le salariat peut intrinsèquement aboutir à une forme d'incertitude que Salais (1989) appelle " incertitude critique sur la productivité". Toutefois, l'approche marchande de Salais est à relativiser au Ferlo où la production animale n'est pas systématiquement commercialisée (Wane et al., 2009). Dans la zone d'enquête, les incertitudes sur la productivité de la main-d'œuvre sont couvertes par des conventions de productivité à travers un processus routinier et répétitif. Rendre visite au berger en grande transhumance relève d'une forme de surveillance et d'évaluation dont les modalités ne sont pas préalablement définies. En dehors des grandes transhumances, l'observation quotidienne du troupeau avant et après la conduite aux pâturages permet de vérifier la production de lait des animaux et l'état général des animaux. Le comportement quotidien du salarié, sa rigueur au travail et ses relations sociales sont également observés. Les incertitudes de l'employeur sur l'équivalence entre "travail futur " et "salaire " sont gérées dans un climat de confiance relative et de contrôles réguliers des efforts fournis par le berger. Le clivage radical entre la confiance en termes de capital cumulatif et la confiance indissociable d'une réalité sociale et collective (Reynaud, 1998), n'est pas si probant dans le contexte du Ferlo. Le recours à de la main-d'œuvre salariée demeure une optimisation opportuniste de la répartition des tâches : pour ceux qui en ont les moyens, on délègue les tâches répétitives - sous contrôle quotidien - à des salariés - recrutés sous contrôle ethnico social - pour se consacrer à d'autres activités - plus lucratives, plus importantes. La confiance, souvent mise en avant par les éleveurs employeurs, n'est pas acquise d'emblée. La proximité géographique joue, avec une confiance prioritairement accordée aux salariés originaires de la même localité que les employeurs (43\% des salariés). La proximité ethnique intervient aussi, surtout près des sites urbains où le choix est plus large. En moyenne, $78 \%$ des employeurs recrutent des salariés d'ethnie peule et $41 \%$ des employeurs ciblent leur propre fraction ${ }^{5}$, avec un pourcentage plus élevé à Boulal, près de la capitale régionale de Dahra (83\% des salariés sont de même localité et ethnie

\footnotetext{
${ }^{4}$ Selon Salais (1989), deux types d'équivalences peuvent être discernés: entre, d'un côté, le " travail futur " et le " salaire " et, de l'autre, le " travail actuel » et le " produit". Ces deux équivalences aboutissent à une "incertitude critique " sur la productivité, se traduisant par l'impossibilité de prédéfinir les comportements des deux parties contractantes de façon complète et en termes économiques.

${ }^{5}$ Cette terminologie est empruntée à Dupire (1996, p. 282) pour qui la fraction dérive d'une première ou d'une seconde segmentation du lignage primaire.
}

et $67 \%$ de même fraction que leurs employeurs). Le climat de confiance s'instaure aussi à travers les relations extraprofessionnelles entre employeurs et salariés; ces derniers sont généralement considérés comme des membres de la famille de l'employeur.

\section{Conclusion}

L'étude du recours à de la main-d'œuvre salariée au Ferlo modifie la représentation habituelle de l'élevage familial pastoral. Le salariat concerne $25 \%$ des exploitations du Ferlo à travers des contrats informels, fondés sur un réseau de connaissances maîtrisé. Cet élevage pastoral ne se sécurise pas seulement par ses ressources familiales, mais par des modes de production combinant à la fois le salariat, l'emploi interne et le transfert de maind'œuvre.

Les incertitudes liées à la délégation des tâches sont plus ou moins gérées par les rapports de confiance (recrutement dans la sphère ethnique, géographique et fractionnelle) et le contrôle des efforts du salarié (accompagnement familial, observations quotidiennes).

\section{Références}

Ancey V, Monas G. Le pastoralisme au Sénégal entre politique moderne et gestion des risques par les pasteurs. Revue Tiers Monde 2005; XLVI, 184: 761-83. www.armand-colin.com/ revues_article_info.php?idr=30 $35:$ 493-518.

Charney J, Stone PH, Quirk WJ. Drought in the Sahara: a biogeophysical feedback mechanism. Science $1975 ; 187$ : 434-5.

Chayanov A. Die Lehre von der bauerlichen Wirtchaft. Berlin: Parey, 1923 (traduction française: L'organisation de l'économie paysanne. Paris: Librairie du Regard, 1990).

Corsi A, Findeis J. True state dependence and heterogeneity in off-farm labor participation. Eur Rev Agric Econ 2000 ; 27 : 127-52.

Dupire M. Peuls nomades. Étude descriptive des Wodaabe du Sahel nigérien. Paris : éditions Karthala, 1996.

Gasson R, Crow G, Errington A, Hutson J, Marsden $T$, Winter $M$. The farm as a familly business : A review. J Agr Econ 1988; 39 : 1-41.

Hair Jr JF, Anderson RE, Tatham RL, Black WC. Multivariate data analysis. New Jersey: Prentice Hall, 1998.

Kimhi A. Family composition and off-farm participation decisions in Israeli farm households. Working Paper No. 15.01. Rehovot (Israel): The Center for Agricultural Economic Research, 2001. http://ageconsearch.umn.edu/bitstream/14976/1/ dp0115.pdf

Lass DA, Gempesaw CM. The supply of off-farm labour: A random coefficients approach. Am J Agr Econ 1992 ; 74 : 400-11. 
Marshall F, Hildebrand E. Cattle before crops: the beginnings of food production in Africa. $J$ World Prehist $2002 ; 16: 99-143$.

Mishra AK, Sandretto CL. Stability of farm income and the role of nonfarm income in US agriculture. Rev Agric Econ 2002 ; 24 : 208-21.

Reardon T, Delgado C, Malton P. Determinants and effects of income diversification among farm households in Burkina Faso. J Dev Stud 1992 ; 28 : 264-96.

Reynaud B. Les conditions de la confiance. Réflexions à partir du rapport salarial. Rev Econ $1998 ; 49: 1455-72$

Salais R. L'analyse économique des conventions du travail. Rev Econ 1989; 40 : 199-240.
Scoones I. Nouvelles orientations du développement pastoral en Afrique. Vivre dans un environnement incertain. Londres: éditions KarthalaCTA, 1999.

Vanderman A, Sadoulet E, de Janvry A. Labor contracting and a theory of contrat choice in California Agriculture. Am J Agr Econ 1991 ; 73 : 681-92.

Vergara O, Coble KH, Patrick GF, Knight TO, Baquet $\mathrm{AE}$. Farm income variability and the supply of off-farm labor by limited-resource farmers. Journal of Agricultural and Applied Economics 2004 ; 39 : 467-79. http://ageconsearch umn.edu/bitstream/43394/2/Vergara\%20JAAE\% 20August\%202004.pdf.
Wane A, Camara AD, Ancey V, Joly N, Kâ SN. Choix individuel et sécurisation collective. Le salariat dans les exploitations pastorales du Ferlo (Sahel sénégalais). Economies et Sociétés, Sér Socio-Economie du Travail, $A B$ 2009; 9 : 1443-68.

Wane A, Ancey V, Touré I. Pastoralisme et recours aux marchés: cas du Sahel sénégalais (Ferlo). Cah Agric 2010; 19: 14-20. doi : 10.1684/agr.2009.0329

Woldehanna T, Oskam A. Income diversification and entry barriers: evidence from the Tigray region of northern Ethiopia. Food Policy 2001; $26: 351-65$. 